\title{
Photoinduced Synthesis of New Diisochromenochromen-4-ones and Their Antimicrobial Activities
}

\author{
Mohamad Yusuf, Indu Solanki, and Payal Jain \\ Department of Chemistry, Punjabi University, Punjab, Patiala147002, India \\ Correspondence should be addressed to Mohamad Yusuf, yusuf_sah04@yahoo.co.in
}

Received 31 October 2011; Accepted 5 December 2011

Academic Editors: R. Dalpozzo and G. Sommen

Copyright ( $) 2012$ Mohamad Yusuf et al. This is an open access article distributed under the Creative Commons Attribution License, which permits unrestricted use, distribution, and reproduction in any medium, provided the original work is properly cited.

\begin{abstract}
The diisochromenochromen-4-one $\mathbf{3 a - 3} \mathbf{b}, \mathbf{4 a - 4}, \mathbf{c}, \mathbf{5} \mathbf{a}-\mathbf{6 a} \& \mathbf{2} 7$ have been prepared from the photocyclization reaction of bischromen4-one 2a-2e. The later compounds are obtained from the O-alkylation of the suitable 3-hydroxy-2-aryl-4H-chromen-4-one 1a1e with 4,4'-bischloromethyl-diphenyl in dry acetone, anhydrous $\mathrm{K}_{2} \mathrm{CO}_{3}$, and $\mathrm{PTC}\left(\mathrm{Bu}_{4} \mathrm{~N}^{+} \mathrm{I}^{-}\right)$under refluxing conditions. The

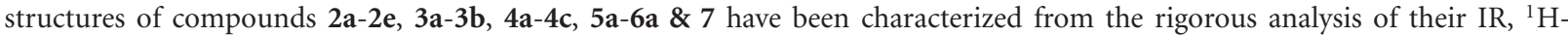
NMR, ${ }^{13} \mathrm{C}-\mathrm{NMR}$, ESI-Mass, and elemental analysis. The antibacterial and antifungal activities of the synthesized products were also evaluated against the Klebsiella pneumoniae, Pseudomonas aeruginosa, Escherichia coli, Staphylococcus aureus, Bacillus subtilis, and Aspergillus janus and Penicillium glabrum, respectively. Some of the tested compounds showed significant activity against the above-said microorganisms.
\end{abstract}

\section{Introduction}

Syntheses of six membered heterocyclic compounds have been the subject of major interest for researchers due to their significant biological activities [1-12]. The photochemical reaction of $\mathrm{C}=\mathrm{O}$ compounds leads to the formation of many exotic carbocyclic and heterocyclic compounds, and these reactions are initiated through the intramolecular $\mathrm{H}$-abstraction by the photoexcited carbonyl group from the $\gamma$ and $\delta$ position to give 1,4 and 1, 5-biradicals which finally collapse to the generation of many unique heterocyclic products [13-20]. 2-Aryl-3-alkoxy-chromen-4one is such substrates which can undergo $\gamma \mathrm{H}$-abstraction to provide pyran derivatives from the cyclisation reaction of 1, 4-biradical and 2-aryl ring [21-23]. The bischromen4-one is the bichromophoric molecules which are formed by joining two chromen-4-one moieties together through the carbon chain of varying length and structure, and photochemical reaction of these compounds may produce some interesting heterocyclic compounds. Generally, the heterocyclic compounds are obtained via multistage reaction in the presence of specific reagents and reaction conditions. We are aiming hereby to synthesize bispyran derivatives under the influence of light. This aspect has prompted us to investigate the synthesis of diisochromeno-chromen-4-ones from the photochemical reaction of bischromen-4-one $2 \mathrm{a}-2 \mathrm{e}$ built around the diphenyl spacer moiety. The major interest behind this study was to investigate the simple method and antimicrobial evaluations of new diisochromeno-chromen4-ones.

\section{Results and Discussion}

The compound $\mathbf{2 a - 2 e}$ required for this study was obtained from the O-alkylation of the suitable 3-hydroxy-chromen4-one [24-26] with 4,4'-bischloromethyldiphenyl in the presence of anhydrous $\mathrm{K}_{2} \mathrm{CO}_{3}$ and $\mathrm{Bu}_{4} \mathrm{~N}^{+} \mathrm{I}^{-}$(PTC) in dry acetone (Scheme 1). The reactions carried out in the absence of PTC provided very poor yield of the bischromen4 -ones and also involved very long reaction times. The monoalkoxy chromen-4-one formed in these reactions was removed by using column chromatography (60-120 mesh). The structures of $\mathbf{2 a - 2 e}$ were determined by means of their IR, ${ }^{1} \mathrm{H}-\mathrm{NMR}$, and ESI-MS spectral data (see the appendix).

The IR spectra of $\mathbf{2 a - 2 e}$ exhibited strong absorptions in the region of $1640-1650 \mathrm{~cm}^{-1}$ which indicated the presence 
<smiles>[Y17]c1oc2ccccc2c(=O)c1OCc1ccc(-c2ccc(COc3c([Al])oc4ccccc4c3=O)cc2)cc1</smiles>

1a-1e $2 a-2 e$<smiles>COc1ccc(I)cc1C(C)(C)C</smiles>

of conjugated $\mathrm{C}=\mathrm{O}$ group. The major feature of their ${ }^{1} \mathrm{H}$ NMR spectra was the appearance of sharp singlet at $\delta 5.11-$ 5.35 which may be assigned to $3-\mathrm{OCH}_{2}$ group. The downfield appearance of this proton could be ascribed to their benzylic nature and placement near an electronegative oxygen atom. ${ }^{13} \mathrm{C}-\mathrm{NMR}$ of these compounds revealed the most significant downfield signal at $\delta$ 176.13-175.01 ( $\mathrm{C}=\mathrm{O}$ group), another signal at $\delta 142.12-139.08$ due to C-3 due of its direct linkage to oxygen atom, and remaining aromatic carbon atoms were resonating at $\delta$ 161.47-117.97. The most upfield resonance was found placed at $\delta 72.07-69.02$ due to $\mathrm{OCH}_{2}$ group.

The photochemical reaction of bischromen-4-ones $2 \mathrm{a}$ 2e was carried out under inert atmosphere in dry $\mathrm{MeOH}$ and THF (1:1) with Pyrex-filtered light from a $125 \mathrm{~W} \mathrm{Hg}$ arc lamp. The progress of the photoreactions was monitored by TLC, and after about $8-10 \mathrm{hrs}$ most of the starting compound was transformed to new products (Scheme 2). The column chromatographic separation of the reaction mixtures yielded $\mathbf{3 a - 3} \mathbf{b}, \mathbf{4 a - 4 c}, \mathbf{5 a}, \mathbf{6} \mathbf{a}$, and 7 in moderate yields. The structure of these products became evident from the comparison of their IR, ${ }^{1} \mathrm{H}-\mathrm{NMR}$, and ${ }^{13} \mathrm{C}-\mathrm{NMR}$ spectra with those of starting compound $2 \mathbf{a}-2 \mathbf{e}$ (see the appendix).

The appearance of one IR absorption band in the carbonyl group region of $1630-1650 \mathrm{~cm}^{-1}$ indicates that these products are obtained involving the both side cyclization on the bischromen-4-one. The ${ }^{1} \mathrm{H}$-NMR spectra of $\mathbf{3 a}$ and $\mathbf{3 b}$ were quite informative which gave broad singlet at $\delta 6.81-$ $6.78(\mathrm{H}-1)$, dd at $\delta 5.85-5.80(\mathrm{H}-3)$, and another dd at $\delta$ 5.30-5.25 (H-4). The noticeable signals were centered at $\delta$ 5.01-5.05 (H-5) and 3.40-3.45 (H-4a) having coupling value of $11.0 \mathrm{~Hz}$ which describes their cis disposition at C-4a and C-5. The four-proton multiplet found at $\delta$ 2.95-2.92 may be assigned to $\mathrm{H}-2 \mathrm{a} .{ }^{13} \mathrm{C}-\mathrm{NMR}$ spectra of these compounds were also very helpful which showed resonances at $\delta 178.20$ 177.82 due to carbonyl group, at $\delta 39.00-38.80$ and $\delta 32.00-$ 31.05 due to $\mathrm{C}-4 \mathrm{a}$ and $\mathrm{C}-2$, respectively. Another signal at $\delta$ 126.53 could be assigned to C-1 which was absent in their starting compounds.

The ${ }^{1} \mathrm{H}-\mathrm{NMR}$ spectrum of $\mathbf{4 a - 4} \mathbf{c}$ was very simple which produced most of the resonances in aromatic region at $\delta 8.30-6.95$, and a significant singlet at $\delta 5.85-5.78(2 \mathrm{H})$ may be allotted to $\mathrm{H}-5 .{ }^{13} \mathrm{C}-\mathrm{NMR}$ of these compounds showed the suitable signals due to aromatic carbon in the region of $\delta$ 156.68-114.58, and the signals at $\delta 72.93-71.74$ could be resulted by $\mathrm{C}-5$.

Similarly the major features of ${ }^{1} \mathrm{H}-\mathrm{NMR}$ spectra of $\mathbf{5 a}$ 6a were the signals due to $\mathrm{H}-3 \mathrm{a}, \mathrm{H}-11 \mathrm{~b}, \& \mathrm{H}-4$ which clearly resonating at $\delta 3.70-3.67(2 \mathrm{H}$, ddd $), 5.11-5.06(2 \mathrm{H}, \mathrm{d})$, and $5.48-5.45(2 \mathrm{H}, \mathrm{d})$, respectively. The coupling value of $J_{3 \mathrm{a}, 11 \mathrm{~b}}=$ $8.1 \mathrm{~Hz}$ and $J_{3 \mathrm{a}, 4}=10.0 \mathrm{~Hz}$ describes the cis relationship both between $\mathrm{H}-3 \mathrm{a} \& \mathrm{H}-11 \mathrm{~b}$ and $\mathrm{H}-3 \mathrm{a} \& \mathrm{H}-4$. In the ${ }^{13} \mathrm{C}-$ NMR spectra, suitable resonances were found to be placed at $\delta$ 178.20-177.62, 83.30-82.10, 47.20-47.12 and 34.50-33.48 which may be very well represented by $\mathrm{C}=\mathrm{O}, \mathrm{C}-4, \mathrm{C}-3 \mathrm{a}$, and $\mathrm{C}-11 \mathrm{~b}$, respectively. The various spectral data also fully confirmed the structural features of the compound 7.

Mechanistically, the phototransformation of bischromen-4-ones 2a-2c may be occurring through the $\mathrm{H}$ abstraction by the photoexcited $\mathrm{C}=\mathrm{O}$ group from the 3 benzyloxy group to give 1,4-biradical which undergoes cyclization with the 2-aryl ring to provide 1,7-biradical. The later may suffer $[1,7]-\mathrm{H}$ shift to give $\mathbf{3} \mathbf{a}-\mathbf{3} \mathbf{b}$ while oxidation of the biradical produces $4 \mathbf{a}-4 \mathbf{c}$ (Scheme 3). Similarly, the formation of the products $5 \mathbf{a}-\mathbf{6} \mathbf{a}$ and 7 from the photocyclization of bischromon-4-ones $\mathbf{2 c - 2 d}$ may also be described.

\section{Antimicrobial Activity}

The antimicrobial activity of synthesized compounds was screened in vitro against selected pathogens which include Staphylococcus aureus (MTCC 96), Bacillus subtilis (MTCC 441), Escherichia coli (MTCC 443), Pseudomonas aeruginosa (MTCC 424), and Klebsiella pneumoniae (MTCC 3384), and fungus strains were Aspergillus janus (2751) and Penicillium glabrum (4951). All the compounds were also screened for MIC by using serial tube dilution method [27] at concentration $3.12,6.25,12.5,25,50$, and $100 \mu \mathrm{g} / \mathrm{mL}$ against the abovesaid microorganisms, and observed minimum inhibitory concentration (MIC- $\mu \mathrm{g} / \mathrm{mL}$ ) values are given in Tables 1 and 2. Compounds $\mathbf{2 a}, \mathbf{2 d}$, and $2 \mathrm{e}$ showed significant activity against Escherichia coli and Klebsiella pneumoniae whereas 


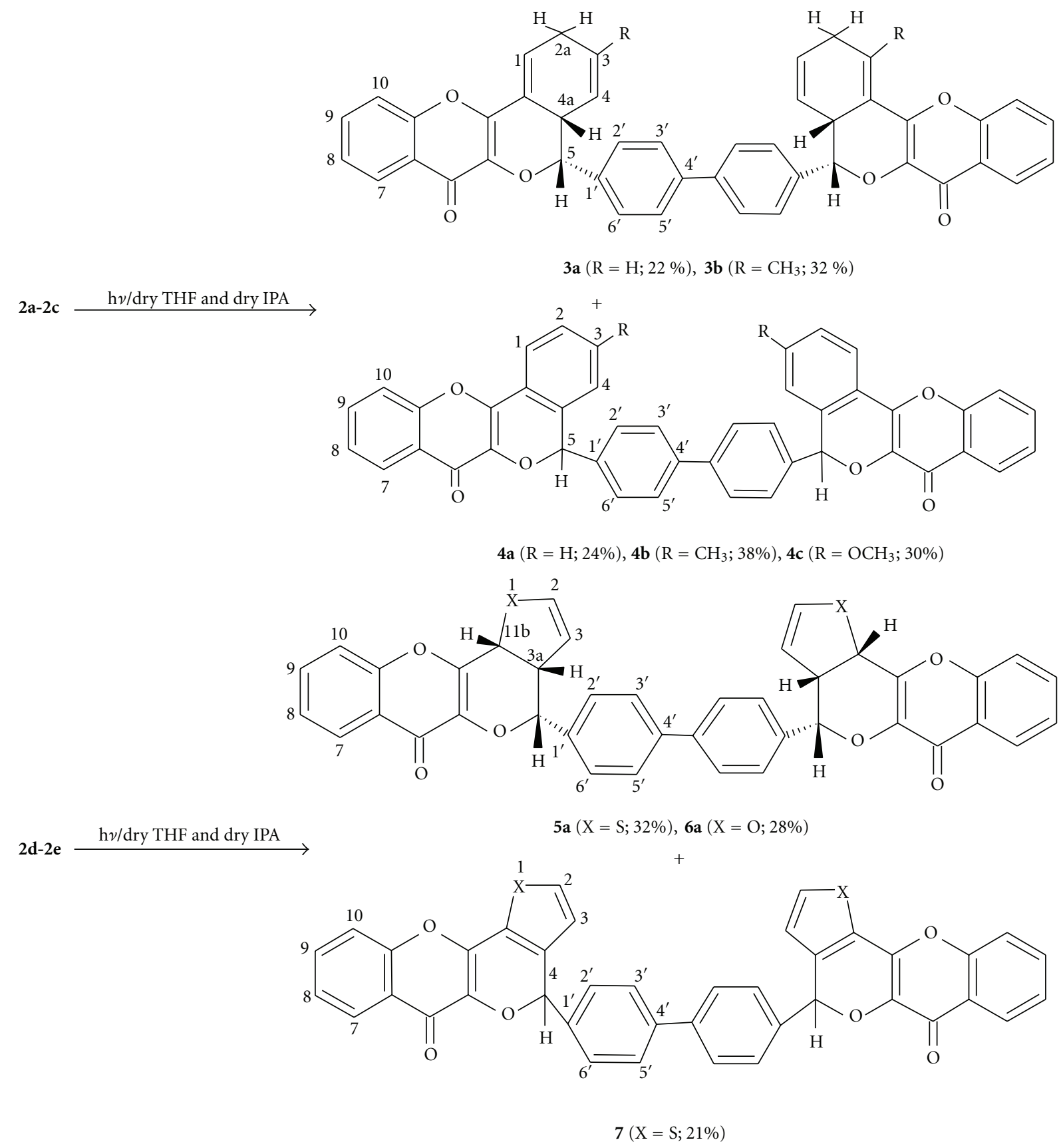

Scheme 2

$\mathbf{2 b}$ and $2 \mathbf{c}$ showed significant activity against Pseudomonas aeruginosa, Staphylococcus aureus, and Pencilluim glabrum. The photoproducts $\mathbf{3} \mathbf{a}, \mathbf{3 b}, \mathbf{4} \mathbf{a}, \mathbf{5} \mathbf{a}$, and $\mathbf{6 a}$ also exhibited significant activity against Escherichia coli, Klebsiella pneumoniae, Pseudomonas aeruginosa, Staphylococcus aureus, and Pencilluim glabrum, respectively. It is evident from the above study that 2-phenyl/thienyl/furanyl-bischromen4-ones seem to be better antimicrobial agents than 2tolyl/anisyl-bischromen-4-one derivatives. The importance of this work lies in the possibility that newly prepared compounds (2a, 2b, 2c, 2d, 3a, 3b, 4a, 5a, and 6a) might be more efficacious derivatives against the above-said bacterial and fungal strains.

\section{Conclusion}

This study provides the photochemical method for the preparation of new diisochromenochromen-4-one linked through the diphenyl moiety. The products have been obtained without using any specific and toxic reagent. The antimicrobial analysis of the prepared compounds has also been carried out and the importance of this work lies in 
<smiles>[R]c1ccc(-c2oc3ccccc3c(=O)c2OCc2ccc(I)cc2)cc1</smiles><smiles>[R]C1=CC2C(=C1)c1oc3ccccc3c(=O)c1[C@H](c1ccc(I)cc1)O[C@H]2c1ccc(I)cc1</smiles>

Scheme 3: Mechanism of the photocyclizations of bischromon-4-ones 2a-2c.

the possibility that newly synthesized compounds $(\mathbf{2 a}, \mathbf{2} \mathbf{b}$, 2c, 2d, 3a, 3b, 4a, 5a, and 6a) might be more efficacious derivatives against the tested bacterial and fungal strains. The investigations regarding the more biological studies of these bischromen-4-ones could be helpful in designing the potent antimicrobial agents.

\section{Appendix}

\section{A. Experimental}

A.1. Synthesis of 3,3'-(Biphenyl-4,4'-Diylbis(Methylene))Bis (Oxy)Bis(2-Phenyl-4H-Chromen-4-One) 2a. The bischromenone $\mathbf{2} \mathbf{a}$ was synthesized by reacting compound $\mathbf{1 a}(2.00 \mathrm{~g}$, 0.0089 mole) with $4,4^{\prime}$-bischloromethyl-diphenyl (1.12 g, 0.0044 mole) in presence of anhyd. $\mathrm{K}_{2} \mathrm{CO}_{3}$ and $\mathrm{Bu}_{4} \mathrm{~N}^{+} \mathrm{I}^{-}$ as PTC in dry acetone. Progress of reaction was monitored by TLC. After the completion of reaction, reaction mixture was turned into colorless mass which was poured into iced $\mathrm{HCl}$ to obtain a crude product which was recrystallized with $\mathrm{CHCl}_{3}: \mathrm{MeOH}$ to yield pure bischromone $\mathbf{2 a}$.

Colorless solid; yield $32 \%$; m.p.: $180-182^{\circ} \mathrm{C}$; IR $(\mathrm{KBr})$ $\mathrm{cm}^{-1}: 1640$ (C=O), 1616 (C=C); ${ }^{1} \mathrm{H}-\mathrm{NMR}$ (400 MHz,
DMSO- $\left.\mathrm{d}_{6}\right): \delta 8.32\left(2 \mathrm{H}, \mathrm{dd}, J_{\mathrm{p}, \mathrm{o}}=1.6,8.0 \mathrm{~Hz}, \mathrm{H}-5\right), 7.99$ $\left(4 \mathrm{H}, \mathrm{dd}, J_{\mathrm{o}}=1.5,5.8 \mathrm{~Hz}, \mathrm{H}-2^{\prime}, 6^{\prime}\right), 7.69(2 \mathrm{H}, \mathrm{m}, \mathrm{H}-7), 7.53$ $\left(2 \mathrm{H}, \mathrm{d}, J_{\mathrm{o}}=8.3 \mathrm{~Hz}, \mathrm{H}-6\right), 7.46\left(12 \mathrm{H}, \mathrm{m}, \mathrm{H}-8,4^{\prime}, 2^{\prime \prime}, 3^{\prime \prime}, 5^{\prime \prime}\right.$, $\left.6^{\prime \prime}\right), 7.34\left(4 \mathrm{H}, \mathrm{d}, J_{\mathrm{o}}=8.3 \mathrm{~Hz}, \mathrm{H}-3^{\prime}, 5^{\prime}\right), 5.16\left(4 \mathrm{H}, \mathrm{s}, \mathrm{OCH}_{2}\right)$; ${ }^{13} \mathrm{C}-\mathrm{NMR}$ (DMSO-d $\left.\mathrm{d}_{6}\right): \delta 176.04(\mathrm{C}-4), 155.23(\mathrm{C}-2), 153.02$ (C-8a), 139.08 (C-3), $139.00\left(\mathrm{C}-4^{\prime \prime}\right), 136.04\left(\mathrm{C}-1^{\prime \prime}\right), 135.82$ (C-7), $130.30\left(\mathrm{C}-1^{\prime}\right), 128.51\left(\mathrm{C}-3^{\prime}, 5^{\prime}\right), 128.10\left(\mathrm{C}-3^{\prime \prime}, 5^{\prime \prime}\right)$, $127.9\left(\mathrm{C}-2^{\prime}, 4^{\prime}, 6^{\prime}\right), 127.6\left(\mathrm{C}-2^{\prime \prime}, 6^{\prime \prime}\right), 125.71(\mathrm{C}-4 \mathrm{a}), 124.21$ (C-5), 123.42 (C-6), 113.64 (C-8), $72.02\left(\mathrm{OCH}_{2}\right)$; MS (ESI): $\mathrm{m} / \mathrm{z}(\mathrm{M})^{+}=655$; Anal. Calc. For $\mathrm{C}_{44} \mathrm{H}_{30} \mathrm{O}_{6}: \mathrm{C}, 80.73 ; \mathrm{H}$, 4.58; found C, $80.69 ; \mathrm{H}, 4.57 \%$.

A.2. Synthesis of 3,3'-(Biphenyl-4,4'-Diylbis(Methylene))Bis (Oxy)Bis(2-p-Tolyl-4H-Chromen-4-One) 2b. The bischromenone $\mathbf{2} \mathbf{b}$ was obtained from the reaction of compound $1 \mathbf{b}(2.00 \mathrm{~g}, 0.0079 \mathrm{~mol})$ with $4,4^{\prime}$-bischloromethyl-diphenyl $(0.99 \mathrm{~g}, 0.0039 \mathrm{~mole})$ under similar condition as described above for $\mathbf{2 a}$.

Colorless solid; yield $24 \%$; m.p.: $208-210^{\circ} \mathrm{C}$; IR $(\mathrm{KBr})$ $\mathrm{cm}^{-1}$ : $1642(\mathrm{C}=\mathrm{O}), 1611 \quad(\mathrm{C}=\mathrm{C})$; ${ }^{1} \mathrm{H}-\mathrm{NMR} \quad(400 \mathrm{MHz}$, DMSO- $\left.\mathrm{d}_{6}\right): \delta 8.30\left(2 \mathrm{H}, \mathrm{dd}, J_{\mathrm{p} . \mathrm{m}}=1.4,7.9 \mathrm{~Hz}, \mathrm{H}-5\right), 7.93$ $\left(4 \mathrm{H}, \mathrm{d}, J_{\mathrm{o}}=8.3 \mathrm{~Hz}, \mathrm{H}-2^{\prime}, 6^{\prime}\right), 7.66 \mathrm{~Hz}\left(2 \mathrm{H}, \mathrm{ddd}, J_{\text {p.m.o. }}=0.9\right.$, $1.6,8.6 \mathrm{~Hz}, \mathrm{H}-7), 7.51\left(2 \mathrm{H}, \mathrm{d}, J_{\mathrm{o}}=8.4 \mathrm{~Hz}, \mathrm{H}-6\right), 7.45(2 \mathrm{H}, \mathrm{d}$, 
TABLE 1: In vitro antimicrobial MIC $(\mu \mathrm{g} / \mathrm{mL})$ of compounds $\mathbf{2 a}-\mathbf{2} \mathbf{b}$.

\begin{tabular}{|c|c|c|c|c|c|c|c|}
\hline \multirow{2}{*}{ Compound No. } & \multicolumn{3}{|c|}{ Gram-negative bacteria } & \multicolumn{2}{|c|}{ Gram-positive bacteria } & \multicolumn{2}{|c|}{ Fungi } \\
\hline & $\begin{array}{c}\text { Escherichia } \\
\text { coli }\end{array}$ & $\begin{array}{l}\text { Klebsiella } \\
\text { pneumonia }\end{array}$ & $\begin{array}{c}\text { Pseudomonas } \\
\text { aeruginosa }\end{array}$ & $\begin{array}{c}\text { Staphylococcus } \\
\text { aureus }\end{array}$ & Bacillus subtilis & Aspergillus janus & $\begin{array}{l}\text { Penicillium } \\
\text { glabrum }\end{array}$ \\
\hline $2 a$ & 12.5 & 12.5 & 50 & 25 & 25 & 50 & 25 \\
\hline $2 b$ & 50 & 25 & 12.5 & 25 & 12.5 & 25 & 50 \\
\hline $2 c$ & 25 & 25 & 12.5 & 12.5 & 25 & 25 & 12.5 \\
\hline $2 d$ & 12.5 & 12.5 & 25 & 25 & 50 & 12.5 & 12.5 \\
\hline $2 \mathrm{e}$ & 12.5 & 12.5 & 12.5 & 25 & 25 & 25 & 12.5 \\
\hline Amoxicillin & 3.12 & 3.12 & 3.12 & 3.12 & 3.12 & & \\
\hline Fluconozole & - & - & - & - & - & 3.12 & 3.12 \\
\hline
\end{tabular}

TABLE 2: In vitro antimicrobial MIC ( $\mu \mathrm{g} / \mathrm{mL})$ of photoinduced compounds 3a-3b, 4a-4c, 5a-6a \& 7 .

\begin{tabular}{|c|c|c|c|c|c|c|c|}
\hline \multirow{2}{*}{ Compound No. } & \multicolumn{3}{|c|}{ Gram-negative bacteria } & \multicolumn{2}{|c|}{ Gram-positive bacteria } & \multicolumn{2}{|c|}{ Fungi } \\
\hline & $\begin{array}{c}\text { Eschericha } \\
\text { coli }\end{array}$ & $\begin{array}{c}\text { Klebsiella } \\
\text { pneumonia }\end{array}$ & $\begin{array}{c}\text { Pseudomonas } \\
\text { aeruginosa }\end{array}$ & $\begin{array}{c}\text { Staphylococcus } \\
\text { aureus }\end{array}$ & $\begin{array}{c}\text { Bacillius } \\
\text { subtilis }\end{array}$ & Aspergillus janus & $\begin{array}{l}\text { Penicillium } \\
\text { glabrum }\end{array}$ \\
\hline $3 a$ & 12.5 & 12.5 & 50 & 12.5 & 50 & 25 & 12.5 \\
\hline $3 b$ & 25 & 25 & 25 & 25 & 12.5 & 12.5 & 12.5 \\
\hline $4 a$ & 12.5 & 50 & 12.5 & 12.5 & 12.5 & 12.5 & 12.5 \\
\hline $4 b$ & 50 & 25 & 25 & 50 & 25 & 50 & 25 \\
\hline $4 c$ & 25 & 12.5 & 25 & 25 & 25 & 12.5 & 50 \\
\hline $5 a$ & 12.5 & 25 & 12.5 & 25 & 25 & 25 & 50 \\
\hline $6 a$ & 50 & 12.5 & 25 & 12.5 & 12.5 & 12.5 & 12.5 \\
\hline 7 & 12.5 & 25 & 50 & 25 & 25 & 25 & 12.5 \\
\hline Amoxicillin & 3.12 & 3.12 & 3.12 & 3.12 & 3.12 & & \\
\hline Fluconozole & - & - & - & - & - & 3.12 & 3.12 \\
\hline
\end{tabular}

$\left.J_{\mathrm{o}}=8.2 \mathrm{~Hz}, \mathrm{H}-8\right), 7.40\left(8 \mathrm{H}, \mathrm{m}, \mathrm{H}-2^{\prime \prime}, 3^{\prime \prime}, 5^{\prime \prime}, 6^{\prime \prime}\right), 7.27(4 \mathrm{H}$, d, $\left.J_{\mathrm{o}}=8.2 \mathrm{~Hz}, \mathrm{H}-3^{\prime}, 5^{\prime}\right), 5.12\left(4 \mathrm{H}, \mathrm{s}, 3-\mathrm{OCH}_{2}\right), 2.44(3 \mathrm{H}, \mathrm{s}$, $\left.4^{\prime}-\mathrm{CH}_{3}\right) ;{ }^{13} \mathrm{C}-\mathrm{NMR}\left(\mathrm{DMSO}-\mathrm{d}_{6}\right): \delta 175.16(\mathrm{C}-4), 156.78(\mathrm{C}-$ 8a), 155.32 (C-2), 141.13 (C-3), 140.61 (C-4'), 139.62 (C$\left.4^{\prime \prime}\right), 135.81\left(\mathrm{C}-1^{\prime \prime}\right), 133.40$ (C-7), 129.32 (C-3', 5'), 129.08 $\left(\mathrm{C}-1^{\prime}\right), 128.80\left(\mathrm{C}-3^{\prime \prime}, 5^{\prime \prime}\right), 128.18\left(\mathrm{C}-2^{\prime \prime}, 6^{\prime \prime}\right), 126.92\left(\mathrm{C}-2^{\prime}\right.$, $\left.6^{\prime}\right), 125.81$ (C-5), 124.69 (C-6), 124.24 (C-4a), 118.06 (C8), $73.71\left(\mathrm{OCH}_{2}\right), 21.61\left(4^{\prime}-\mathrm{CH}_{3}\right) ; \mathrm{MS}(\mathrm{ESI}): \mathrm{m} / \mathrm{z}(\mathrm{M})^{+}=$ 683; Anal. Calc. For $\mathrm{C}_{46} \mathrm{H}_{34} \mathrm{O}_{6}$ : C, 80.93; H, 4.98\%; found C, 80.86; H, 4.97\%.

A.3. Synthesis of 3,3'-(Biphenyl-4,4'-Diylbis(Methylene))Bis (Oxy)Bis(2-(4-Methoxyphenyl)-4H-Chroman-4-One) $2 c$. The bischromenone $2 \mathrm{c}$ was synthesized from the reaction of compound 1c ( $2.00 \mathrm{~g}, 0.0074$ mole) with 4,4' -bis-chloromethyldiphenyl (0.93 g, 0.0037 mole) under similar condition as described above for $2 \mathbf{a}$.

Colorless solid; yield 36\%; m.p.: $194-196^{\circ} \mathrm{C}$; IR(KBr) $\mathrm{cm}^{-1}: 1638$ (C=O), $1603 \quad(\mathrm{C}=\mathrm{C}){ }^{1} \mathrm{H}-\mathrm{NMR} \quad(400 \mathrm{MHz}$, DMSO-d 6$): \delta 8.20\left(2 \mathrm{H}, \mathrm{dd}, J_{\text {p.o }}=1.4,7.9 \mathrm{~Hz}, \mathrm{H}-5\right), 8.04(4 \mathrm{H}$, dd, $\left.J_{\mathrm{p}, \mathrm{o}}=1.8,7.0 \mathrm{~Hz}, \mathrm{H}-2^{\prime}, 6^{\prime}\right), 7.74\left(2 \mathrm{H}\right.$, ddd, $J_{\text {p.m.o }}=1.0$, 2.0, 7.6 Hz, H-7), $7.60\left(2 \mathrm{H}, \mathrm{d}, J_{\mathrm{o}}=8.3 \mathrm{~Hz}, \mathrm{H}-6\right), 7.51(4 \mathrm{H}, \mathrm{d}$, $\left.J_{\mathrm{o}}=8.3 \mathrm{~Hz}, \mathrm{H}-3^{\prime \prime}, 5^{\prime \prime}\right), 7.43\left(6 \mathrm{H}, \mathrm{m}, \mathrm{H}-8,2^{\prime \prime}, 6^{\prime \prime}\right), 7.00(4 \mathrm{H}$, $\left.\mathrm{dd}, J_{\mathrm{p}, \mathrm{o}}=1.8,7.0 \mathrm{~Hz}, \mathrm{H}-3^{\prime}, 5^{\prime}\right), 5.11\left(4 \mathrm{H}, \mathrm{s}, 3-\mathrm{OCH}_{2}\right), 3.88$

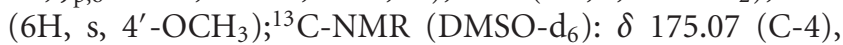

161.47 (C-8a), 156.61 (C-4'), 155.23 (C-2), $140.59\left(\mathrm{C}-1^{\prime \prime}\right)$, 139.18 (C-3), 135.82 (C-7), 133.32 (C-5), 130.62 (C-3", $\left.5^{\prime \prime}\right), 129.36\left(\mathrm{C}-2^{\prime \prime}, 6^{\prime \prime}\right), 126.90\left(\mathrm{C}-2^{\prime}, 6^{\prime}\right), 125.79(\mathrm{C}-4 \mathrm{a})$, 124.66 (C-6), 124.21 (C-4' $\left.{ }^{\prime \prime}\right), 123.37\left(\mathrm{C}-1^{\prime}\right), 117.97\left(\mathrm{C}-3^{\prime}, 5^{\prime}\right)$, $113.75(\mathrm{C}-8), 73.67\left(\mathrm{OCH}_{2}\right), 55.45\left(4^{\prime}-\mathrm{OCH}_{3}\right)$; MS (ESI): $\mathrm{m} / \mathrm{z}(\mathrm{M})^{+}=715$; Anal. Calc. For $\mathrm{C}_{46} \mathrm{H}_{34} \mathrm{O}_{4}$ : C, 77.31\%; H, $4.76 \%$; found $\mathrm{C}, 77.54 ; \mathrm{H}, 4.77 \%$.

A.4. Synthesis of 3,3'-(Biphenyl-4,4'-Diylbis(Methylene))Bis (Oxy)Bis(2-(Thiophen-2-yl)-4H-Chromen-4-One 2d. The bischromenone $2 \mathbf{d}$ was synthesized from the reaction of compound 1d (2.00 g, 0.0087 mole) with 4,4' -bis-chloromethyldiphenyl (1.10 g, 0.0043 mole) under similar condition as described above for $\mathbf{2 a}$.

Colorless solid; yield 33\%; m.p.: $225-227^{\circ} \mathrm{C}$; IR (KBr) $\mathrm{cm}^{-1}$ : 1641 (C=O), 1603 (C=C); ${ }^{1} \mathrm{H}-\mathrm{NMR} \quad(400 \mathrm{MHz}$, DMSO- $\left.\mathrm{d}_{6}\right): \delta 8.22\left(2 \mathrm{H}, \mathrm{dd}, J_{\mathrm{m}, \mathrm{o}}=1.7,8.0 \mathrm{~Hz}, \mathrm{H}-5\right), 7.96$ $\left(2 \mathrm{H}, \mathrm{d}, J_{3^{\prime}, 4^{\prime}}=3.7 \mathrm{~Hz}, \mathrm{H}-3^{\prime}\right), 7.72\left(2 \mathrm{H}, \mathrm{ddd}, J_{\mathrm{p}, \mathrm{m}, \mathrm{o}}=1.2,2.4\right.$, $7.8 \mathrm{~Hz}, \mathrm{H}-7), 7.65\left(2 \mathrm{H}, \mathrm{d}, J_{5^{\prime}, 4^{\prime}}=4.8 \mathrm{~Hz}, \mathrm{H}-5^{\prime}\right), 7.62(4 \mathrm{H}$, $\left.\mathrm{d}, J_{\mathrm{o}}=8.0 \mathrm{~Hz}, \mathrm{H}-3^{\prime \prime}, 5^{\prime \prime}\right), 7.54\left(6 \mathrm{H}, \mathrm{m}, \mathrm{H}-8,2^{\prime \prime}, 6^{\prime \prime}\right), 7.48$ $\left(2 \mathrm{H}, \mathrm{d}, J_{\mathrm{o}}=8.0 \mathrm{~Hz}, \mathrm{H}-6\right), 7.20(2 \mathrm{H}, \mathrm{dd}, J=3.7,4.8 \mathrm{~Hz}, \mathrm{H}-$ $\left.4^{\prime}\right), 5.35\left(4 \mathrm{H}, \mathrm{s}, 3-\mathrm{OCH}_{2}\right),{ }^{13} \mathrm{C}-\mathrm{NMR}\left(\mathrm{DMSO}-\mathrm{d}_{6}\right): \delta 176.13$ (C-4), 157.21 (C-8a), 142.12 (C-3), 140.14 (C-1" ), 136.93 (C-2), $135.52\left(\mathrm{C}-4^{\prime \prime}\right), 135.3$ (C-7), 130.61 (C-5), 130.53 (C$\left.5^{\prime}\right), 129.08\left(\mathrm{C}-2^{\prime}\right), 128.80\left(\mathrm{C}-3^{\prime \prime}, 5^{\prime \prime}\right), 128.21\left(\mathrm{C}-4^{\prime}\right), 128.18$ 
(C-2" $\left.{ }^{\prime \prime}, 6^{\prime \prime}\right), 127.74\left(\mathrm{C}-3^{\prime}\right), 124.00$ (C-4a), 123.24 (C-6), 117.6 (C-8), $69.62\left(\mathrm{OCH}_{2}\right)$; MS (ESI): m/z (M) ${ }^{+}=667$; Anal. Calc. For $\mathrm{C}_{40} \mathrm{H}_{26} \mathrm{O}_{3} \mathrm{~S}$; C, 72.07, H, 3.90, S, 9.61; found C, 72.13, H, 3.91, S, 9.63\%.

A.5. Synthesis of 3,3'-(Biphenyl-4,4'-Diylbis(Methylene))Bis (Oxy)Bis(2-(Furan-2-yl)-4H-Chromen-4-One $2 \boldsymbol{e}$. The bischromenone $2 \mathrm{e}$ was synthesized from the reaction of compound 1c (2.00 g, 0.0082 mole) with 4,4' -bischloromethyldiphenyl (1.02 g, 0.0041 mole) under similar condition as described above for $2 \mathbf{a}$.

Colorless solid; yield 25\%; m.p.: $248-250^{\circ} \mathrm{C}$; IR (KBr) $\mathrm{cm}^{-1}$ : 1640 (C=O), 1605 (C=C); ${ }^{1} \mathrm{H}-\mathrm{NMR} \quad(400 \mathrm{MHz}$, DMSO-d $\left.{ }_{6}\right): \delta 8.20\left(2 \mathrm{H}, \mathrm{dd}, J_{\mathrm{p}, \mathrm{o}}=1.5,8.1 \mathrm{~Hz}, \mathrm{H}-5\right), 7.81$ $\left(2 \mathrm{H}, \mathrm{d}, J_{3^{\prime}, 4^{\prime}}=1.2 \mathrm{~Hz}, \mathrm{H}-3^{\prime}\right), 7.75\left(2 \mathrm{H}\right.$, ddd, $J_{\mathrm{p}, \mathrm{m}, \mathrm{o}}=1.5,2.4$, $7.8 \mathrm{~Hz}, \mathrm{H}-7), 7.62\left(10 \mathrm{H}, \mathrm{m}, \mathrm{H}-8,2^{\prime \prime}, 6^{\prime \prime}, 3^{\prime \prime}, 5^{\prime \prime}\right), 7.45(2 \mathrm{H}$, t, $\left.J_{\mathrm{o}}=8.0 \mathrm{~Hz}, \mathrm{H}-6\right), 7.34\left(2 \mathrm{H}, \mathrm{d}, J_{5^{\prime}, 4^{\prime}}=3.8 \mathrm{~Hz}, \mathrm{H}-5^{\prime}\right), 6.66$ $\left(1 \mathrm{H}, \mathrm{dd}, J_{5^{\prime}, 4^{\prime}}=3.6 \mathrm{~Hz}, \mathrm{H}-4^{\prime}\right), 5.32\left(4 \mathrm{H}, \mathrm{s}, 3-\mathrm{OCH}_{2}\right) ;{ }^{13} \mathrm{C}-$ NMR (DMSO-d 6 ): $\delta 176.01(\mathrm{C}-4), 157.16(\mathrm{C}-8 \mathrm{a}), 141.92(\mathrm{C}-$ 3), $140.16\left(\mathrm{C}-1^{\prime \prime}\right), 138.91$ (C-2), $135.23\left(\mathrm{C}-4^{\prime \prime}\right), 135.11(\mathrm{C}-$ 7), 130.59 (C-5), $130.49\left(\mathrm{C}-5^{\prime}\right), 129.12\left(\mathrm{C}-2^{\prime}\right), 128.81\left(\mathrm{C}-3^{\prime \prime}\right.$, $\left.5^{\prime \prime}\right), 128.23\left(\mathrm{C}-4^{\prime}\right), 128.10\left(\mathrm{C}-2^{\prime \prime}, 6^{\prime \prime}\right), 127.72\left(\mathrm{C}-3^{\prime}\right), 124.02$ (C-4a), 123.12 (C-6), 117.43 (C-8), $69.63\left(\mathrm{OCH}_{2}\right)$; MS (ESI): $\mathrm{m} / \mathrm{z}(\mathrm{M})^{+}=635$; Anal. Calc. For $\mathrm{C}_{40} \mathrm{H}_{26} \mathrm{O}_{8}: \mathrm{C}, 75.70, \mathrm{H}$, 4.10; found C, 75.47, H, 4.08\%.

A.6. Synthesis of (4aS,5R)-5-( $4^{\prime}-((4 a R, 5 S)-7-O x o-2,4 a, 5,7-$ Tetrahydroisochromeno [4,3-b]Chromen-5-yl)Biphenyl-4-yl)4a,5-Dihydroisochromeno [4,3-b]Chromen-7(2H)-One 3a. A deoxygenated solution of bischromone $2 \mathbf{a}$ ( $100 \mathrm{mg}, 0.00015$ mole) was carried out in dry IPA and dry THF with light from a $125 \mathrm{Hg}$ arc lamp under nitrogen atmosphere in a pyrex vessel for 5 hours. The progress of reaction was monitored by TLC. The solvent was distilled out under reduced pressure, and the resulting photolysate thus obtained was subjected to extensive column chromatography (100-200 mesh), packed in petroleum ether-benzene $(1: 1)$. Elution of column with benzene-EtOAc (3:1) furnished starting compound $2 \mathrm{a}(14 \%$, IR, co-tlc and $\mathrm{mmp})$ and two new compounds $\mathbf{3 a}$ and $\mathbf{4} \mathbf{a}$ which were further recrystallized using $\mathrm{MeOH}$.

Pale yellow solid; yield 22\%; m.p.: 222-224 ${ }^{\circ}$; $\mathrm{IR}(\mathrm{KBr}) \mathrm{cm}^{-1}: 1648(\mathrm{C}=\mathrm{O})$; ${ }^{1} \mathrm{H}-\mathrm{NMR}(400 \mathrm{MHz}, \mathrm{DMSO}-$ $\left.\mathrm{d}_{6}\right): \delta 8.30\left(2 \mathrm{H}, \mathrm{dd}, J_{\mathrm{p}, \mathrm{o}}=1.6,8.2 \mathrm{~Hz}, \mathrm{H}-7\right), 7.67(2 \mathrm{H}, \mathrm{m}$, $\mathrm{H}-9), 7.63\left(4 \mathrm{H}, \mathrm{dd}, J_{\mathrm{p}, \mathrm{o}}=1.0,8.5 \mathrm{~Hz}, \mathrm{H}-3^{\prime \prime}, 5^{\prime \prime}\right), 7.60(2 \mathrm{H}$, $\mathrm{m}, \mathrm{H}-10), 7.58\left(4 \mathrm{H}, \mathrm{dd}, J_{\mathrm{p}, \mathrm{o}}=1.0,8.6 \mathrm{~Hz}, \mathrm{H}-2^{\prime \prime}, 6^{\prime \prime}\right), 7.55$ $\left(2 \mathrm{H}, \mathrm{d}, J_{\mathrm{o}}=8.1 \mathrm{~Hz}, \mathrm{H}-8\right), 6.78(2 \mathrm{H}, \mathrm{brs}, \mathrm{H}-1), 5.85(2 \mathrm{H}$, $\left.\mathrm{dd}, J_{3,2 \mathrm{a}}=1.2, J_{3,4}=10.0 \mathrm{~Hz}, \mathrm{H}-3\right), 5.30\left(1 \mathrm{H}, \mathrm{dd}, J_{4,4 \mathrm{a}}=\right.$ 2.0, $\left.J_{4,3}=10.3 \mathrm{~Hz}, \mathrm{H}-4\right), 5.01\left(2 \mathrm{H}, \mathrm{d}, J_{5,4 \mathrm{a}}=11.0 \mathrm{~Hz}, \mathrm{H}-5\right)$, $3.40\left(2 \mathrm{H}, \mathrm{dd}, J_{4 \mathrm{a}, 4}=2.0, J_{4 \mathrm{a}, 5}=11.0 \mathrm{~Hz}, \mathrm{H}-4 \mathrm{a}\right), 2.95(4 \mathrm{H}$, m, H-2a); 13C-NMR (DMSO-d 6 ): $\delta 177.82$ (C-6), 155.52 (C-10a), 142.80 (C-6a), 141.50 (C-11b), 138.70 (C-4" ${ }^{\prime \prime}$, $136.43\left(\mathrm{C}-1^{\prime \prime}\right), 134.82$ (C-9), $129.82(\mathrm{C}-4), 128.10\left(\mathrm{C}-3^{\prime \prime}\right.$, $\left.5^{\prime \prime}\right), 127.62\left(\mathrm{C}-2^{\prime \prime}, 6^{\prime \prime}\right), 126.53(\mathrm{C}-1), 125.78$ (C-7), 126.02 (C-3), 122.80 (C-8), 122.72 (C-11a), 117.02 (C-10), 82.5 (C-5), $38.02(\mathrm{C}-4 \mathrm{a}), 32.00(\mathrm{C}-2 \mathrm{a})$; MS (ESI): $\mathrm{m} / \mathrm{z}(\mathrm{M})^{+}=$ 655; Anal. Calc. For $\mathrm{C}_{44} \mathrm{H}_{30} \mathrm{O}_{6}$ : C, 80.73; H, 4.58\%; C, 80.97; $\mathrm{H}, 4.57 \%$.
A.7. 5,5' -(Biphenyl-4,4'-Diyl)Diisochromeno [4,3-b] Chromen-7(5H)-One 4a. Brown solid; Yield 24\%; m.p.: 218$220^{\circ} \mathrm{C}$; IR $(\mathrm{KBr}) \mathrm{cm}^{-1}$ : $1652(\mathrm{C}=\mathrm{O}),{ }^{1} \mathrm{H}-\mathrm{NMR}(400 \mathrm{MHz}$, DMSO- $\left.\mathrm{d}_{6}\right): \delta 8.30\left(2 \mathrm{H}, \mathrm{dd}, J_{\mathrm{p}, \mathrm{o}}=1.6,8.0 \mathrm{~Hz}, \mathrm{H}-7\right), 7.98(2 \mathrm{H}$, $\left.\mathrm{dd}, J_{\mathrm{m}, \mathrm{o}}=2.4,8.9 \mathrm{~Hz}, \mathrm{H}-1\right), 7.64(2 \mathrm{H}, \mathrm{m}, \mathrm{H}-9), 7.62(4 \mathrm{H}, \mathrm{dd}$, $\left.J_{\mathrm{p}, \mathrm{o}}=1.0,8.5 \mathrm{~Hz}, \mathrm{H}-3^{\prime \prime}, 5^{\prime \prime}\right), 7.59(2 \mathrm{H}, \mathrm{m}, \mathrm{H}-10), 7.57(4 \mathrm{H}$, $\left.\mathrm{dd}, J_{\mathrm{p}, \mathrm{o}}=1.0,8.4 \mathrm{~Hz}, \mathrm{H}-2^{\prime \prime}, 6^{\prime \prime}\right), 7.58\left(2 \mathrm{H}, \mathrm{d}, J_{\mathrm{o}}=8.0 \mathrm{~Hz}, \mathrm{H}-\right.$ 8), $7.48(4 \mathrm{H}, \mathrm{m}, \mathrm{H}-2,3), 7.10\left(2 \mathrm{H}, \mathrm{dd}, J_{\mathrm{m}, \mathrm{o}}=2.4,8.5 \mathrm{~Hz}, \mathrm{H}-\right.$ 4), $5.85(2 \mathrm{H}, \mathrm{s}, \mathrm{H}-5) ;{ }^{13} \mathrm{C}-\mathrm{NMR}\left(\mathrm{DMSO}-\mathrm{d}_{6}\right): \delta 180.02(\mathrm{C}-7)$, 156.62 (C-12a), 156.21 (C-11a), 139.00 (C-4" $\left.4^{\prime \prime}\right), 136.04$ (C$\left.1^{\prime \prime}\right), 134.82$ (C-10), $128.32\left(\mathrm{C}-3^{\prime \prime}, 5^{\prime \prime}\right), 128.23$ (C-1), 128.12 (C-3), 127.93 (C-8), 127.6 (C-2" , 6" ), 127.19 (C-4a), 124.40 (C-4), 123.02 (C-2), 122.98 (C-9), 121.67 (C-6a), 113.68 (C11), 72.93 (C-5); MS (ESI): $\mathrm{m} / \mathrm{z}(\mathrm{M})^{+}=648$; Anal Calc. for $\mathrm{C}_{44} \mathrm{H}_{26} \mathrm{O}_{6}$ : C, 81.23; H, 4.00; found C, 81.26; H, 4.16\%.

A.8. Synthesis of (4aS,5R)-3-Methyl-5-(4'-((4aR,5S)-1-Methyl-7-Oxo-2,4a,5,7-Tetrahydroisochromeno [4,3-b] Chromen5-yl)biphenyl-4-yl)-4a,Dihydroisochromeno-[4,3-b]Chromen$7(2 \mathrm{H})$-One $\mathbf{3} \boldsymbol{b}$. The photoirradiation of $\mathbf{2}$ bwas carried out under similar condition as described above for $2 \mathbf{a}$. The resulting reaction mixture upon chromatographic workup yielded two new photoproducts $\mathbf{3 b}$ and $\mathbf{4 b}$.

Light yellow solid; yield $32 \%$; m.p.: $168-170^{\circ} \mathrm{C}$; $\mathrm{IR}(\mathrm{KBr}) \mathrm{cm}^{-1}$ : $1638(\mathrm{C}=\mathrm{O})$; ${ }^{1} \mathrm{H}-\mathrm{NMR}(400 \mathrm{MHz}, \mathrm{DMSO}-$ $\left.\mathrm{d}_{6}\right): \delta 8.28\left(2 \mathrm{H}, \mathrm{dd}, J_{\mathrm{p}, \mathrm{o}}=1.4,8.1 \mathrm{~Hz}, \mathrm{H}-7\right), 7.65(2 \mathrm{H}, \mathrm{ddd}$, $\left.J_{\mathrm{p}, \mathrm{m}, \mathrm{o}}=1.0,2.6,8.5 \mathrm{~Hz}, \mathrm{H}-9\right), 7.57\left(2 \mathrm{H}, \mathrm{d}, J_{\mathrm{o}}=8.0 \mathrm{~Hz}, \mathrm{H}-8\right)$, $7.54(2 \mathrm{H}, \mathrm{m}, \mathrm{H}-10) 7.53\left(8 \mathrm{H}, \mathrm{m}, \mathrm{H}-2^{\prime \prime}, 3^{\prime \prime}, 5^{\prime \prime}, 6^{\prime \prime}\right), 6.81$ $\left(2 \mathrm{H}\right.$, brs, H-1), $5.25\left(1 \mathrm{H}, \mathrm{dd}, J_{\text {allyl }, 4,4 \mathrm{a}}=1.0,1.9 \mathrm{~Hz}, \mathrm{H}-4\right)$, $5.05\left(2 \mathrm{H}, \mathrm{d}, J_{5,4 \mathrm{a}}=11.0 \mathrm{~Hz}, \mathrm{H}-5\right), 3.45\left(2 \mathrm{H}, \mathrm{dd}, J_{4,4 \mathrm{a}}=\right.$ 1.9, $\left.J_{4 \mathrm{a}, 5}=11.0 \mathrm{~Hz}, \mathrm{H}-4 \mathrm{a}\right), 2.92(4 \mathrm{H}, \mathrm{m}, \mathrm{H}-2 \mathrm{a}), 2.42(3 \mathrm{H}$, s, $\left.4^{\prime}-\mathrm{CH}_{3}\right) ;{ }^{13} \mathrm{C}-\mathrm{NMR}\left(\mathrm{DMSO}-\mathrm{d}_{6}\right): \delta 177.20(\mathrm{C}-6), 157.20$ (C-11), 143.50 (C-11b), 139.70 (C-4" $), 139.52$ (C-1" ${ }^{\prime \prime}$, 135.20 (C-9), 129.90 (C-4), 128.07 (C-3" $\left.5^{\prime \prime}\right), 127.62$ $\left(\mathrm{C}-2^{\prime \prime}, 6^{\prime \prime}\right), 126.53$ (C-1), 125.78 (C-7), 125.10 (C-3), 123.40 (C-8), 122.68 (C-11a), 118.72 (C-10), 81.5 (C-5), 39.02 (C-4a), $20.98\left(3-\mathrm{CH}_{3}\right)$; MS (ESI): $\mathrm{m} / \mathrm{z}(\mathrm{M})^{+}=683$; Anal Calc. For C, 80.93; H, 4.98; found C, 80.89 ; H, 4.96\%.

A.9. 5,5'-(Biphenyl-4,4'-Diyl)Bis(3-Methylisochromeno [4,3b]Chromen-7(5H)-One) $\mathbf{4 b}$. Light brown solid; yield 38\%; m.p.: $179-181^{\circ} \mathrm{C}$; $\operatorname{IR}(\mathrm{KBr}) \mathrm{cm}^{-1}$ : $1638(\mathrm{C}=\mathrm{O}) ;{ }^{1} \mathrm{H}-\mathrm{NMR}$ $\left(400 \mathrm{MHz}, \mathrm{DMSO}-\mathrm{d}_{6}\right): \delta 8.26\left(2 \mathrm{H}, \mathrm{dd}, J_{\mathrm{p}, \mathrm{o}}=1.3,8.2 \mathrm{~Hz}, \mathrm{H}-\right.$ 7), $7.91\left(2 \mathrm{H}, \mathrm{d}, J_{\mathrm{o}}=8.9 \mathrm{~Hz}, \mathrm{H}-1\right), 7.63\left(2 \mathrm{H}\right.$, ddd, $J_{\mathrm{p}, \mathrm{m}, \mathrm{o}}=$ 1.0, 2.5, 8.4 Hz, H-9), $7.55\left(2 \mathrm{H}, \mathrm{d}, J_{o}=8.0 \mathrm{~Hz}, \mathrm{H}-8\right), 7.50$ $(2 \mathrm{H}, \mathrm{m}, \mathrm{H}-10), 7.48\left(8 \mathrm{H}, \mathrm{m}, \mathrm{H}-2^{\prime \prime}, 3^{\prime \prime}, 5^{\prime \prime}, 6^{\prime \prime}\right), 7.08(4 \mathrm{H}$, m, H-2, 4), $5.82(2 \mathrm{H}, \mathrm{s}, \mathrm{H}-5), 2.43\left(3 \mathrm{H}, \mathrm{s}, 4^{\prime}-\mathrm{CH}_{3}\right) ;{ }^{13} \mathrm{C}-\mathrm{NMR}$ $\left(\mathrm{DMSO}-\mathrm{d}_{6}\right): \delta 180.62(\mathrm{C}-7), 156.68(\mathrm{C}-12 \mathrm{a}), 156.24(\mathrm{C}-11 \mathrm{a})$, $139.04\left(\mathrm{C}-4^{\prime \prime}\right), 136.01$ (C-1" $), 134.78$ (C-10), 128.62 (C-3", $\left.5^{\prime \prime}\right), 128.23$ (C-1), 128.01 (C-3), 127.98 (C-8), 127.62 (C$\left.2^{\prime \prime}, 6^{\prime \prime}\right), 127.01$ (C-4a), 124.42 (C-4), 123.08 (C-2), 122.95 (C-9), 121.58 (C-6a), 114.52 (C-11), 72.73 (C-5), 20.98 (3$\mathrm{CH}_{3}$ ); MS (ESI): $\mathrm{m} / \mathrm{z}(\mathrm{M})^{+}=679$; Anal Calc. for $\mathrm{C}_{46} \mathrm{H}_{30} \mathrm{O}_{6}$ : C, $81.41 ; \mathrm{H}, 4.42$; found C, $81.39 ; \mathrm{H}, 4.43 \%$.

A.10. Synthesis of 5,5'-(Biphenyl-4,4'-Diyl)Bis(3-Methoxyisochromeno [4,3-b]Chromen-7(5H)-One 4c. The deoxygenated THF solution of $2 \mathrm{c}$ was photolysed under similar 
conditions as employed for $2 \mathbf{a}$ and the chromatographic separation of photolysate furnished starting compound $2 \mathbf{a}$ and compound $\mathbf{4 c}$.

Yellow solid; yield $30 \%$; m.p.: $240-242^{\circ} \mathrm{C}$; $\mathrm{IR}(\mathrm{KBr}) \mathrm{cm}^{-1}$ : $1642(\mathrm{C}=\mathrm{O}) ;{ }^{1} \mathrm{H}-\mathrm{NMR}\left(400 \mathrm{MHz}, \mathrm{DMSO}-\mathrm{d}_{6}\right): \delta 8.22(2 \mathrm{H}$, dd, $\left.J_{\mathrm{p}, \mathrm{o}}=1.4,7.8 \mathrm{~Hz}, \mathrm{H}-7\right), 7.86\left(2 \mathrm{H}, \mathrm{d}, J_{\mathrm{o}}=8.7 \mathrm{~Hz}, \mathrm{H}-1\right)$, $7.72\left(2 \mathrm{H}, \mathrm{ddd}, J_{\mathrm{p}, \mathrm{m}, \mathrm{o}}=1.0,2.1,8.3 \mathrm{~Hz}, \mathrm{H}-9\right), 7.59(2 \mathrm{H}, \mathrm{d}$, $\left.J_{\mathrm{o}}=8.2 \mathrm{~Hz}, \mathrm{H}-8\right), 7.52\left(8 \mathrm{H}, \mathrm{m}, \mathrm{H}-2^{\prime \prime}, 3^{\prime \prime}, 5^{\prime \prime}, 6^{\prime \prime}\right), 7.49(2 \mathrm{H}$, m, H-10), 6.95 (2H, m, H-2, 4), 5.78 (2H, s, H-5), $3.84(6 \mathrm{H}$, $\left.\mathrm{s}, 4^{\prime}-\mathrm{OCH}_{3}\right) ; 13 \mathrm{C}-\mathrm{NMR}\left(\mathrm{DMSO}-\mathrm{d}_{6}\right): \delta 182.02(\mathrm{C}-6), 156.04$ (C-11, 11a), $139.04\left(\mathrm{C}-4^{\prime \prime}\right), 136.07\left(\mathrm{C}-1^{\prime \prime}\right), 128.4\left(\mathrm{C}-3^{\prime \prime}, 5^{\prime \prime}\right)$, 128.13 (C-1), 127.82 (C-2), 127.52 (C-2" $\left.4^{\prime \prime}\right), 125.80$ (C-3), 124.32 (C-4), 123.52 (C-2), 114.58 (C-11), 71.74 (C-5); MS (ESI): $\mathrm{m} / \mathrm{z}(\mathrm{M})^{+}=711$; Anal Calc. for $\mathrm{C}_{46} \mathrm{H}_{30} \mathrm{O}_{8}$ : C, 77.75; $\mathrm{H}, 4.22$; found $\mathrm{C}, 77.98 ; \mathrm{H}, 4.20 \%$.

A.11. Synthesis of $\mathbf{5 a}$. The photolysis of $\mathbf{2 d}$ under similar conditions as used for $2 \mathbf{a}$ provided starting bischromone $\mathbf{2 a}$ and two new products $\mathbf{5 a}$ and $\mathbf{7}$.

Light yellow solid; yield $32 \%$; m.p.: $218-220^{\circ} \mathrm{C}$; $\mathrm{IR}(\mathrm{KBr}) \mathrm{cm}^{-1}: 1658(\mathrm{C}=\mathrm{O}) ;{ }^{1} \mathrm{H}-\mathrm{NMR}(400 \mathrm{MHz}, \mathrm{DMSO}-$ $\left.\mathrm{d}_{6}\right): \delta 8.20\left(2 \mathrm{H}, \mathrm{dd}, J_{\mathrm{p}, \mathrm{o}}=1.6,8.0 \mathrm{~Hz}, \mathrm{H}-7\right), 7.70(2 \mathrm{H}, \mathrm{ddd}$, $\left.J_{\mathrm{p}, \mathrm{m}, \mathrm{o}}=1.0,2.3,7.9 \mathrm{~Hz}, \mathrm{H}-9\right), 7.62\left(4 \mathrm{H}, \mathrm{d}, J_{\mathrm{o}}=8.0 \mathrm{~Hz}, \mathrm{H}-3^{\prime \prime}\right.$, $\left.5^{\prime \prime}\right), 7.55\left(6 \mathrm{H}, \mathrm{m}, \mathrm{H}-10,2^{\prime \prime}, 6^{\prime \prime}\right), 7.52\left(2 \mathrm{H}, \mathrm{d}, J_{\mathrm{o}}=8.1 \mathrm{~Hz}\right.$, $\mathrm{H}-8), 6.40\left(2 \mathrm{H}, \mathrm{dd}, J_{2,3 \mathrm{a}}=1.0, J_{2,3}=5.8 \mathrm{~Hz}, \mathrm{H}-2\right), 5.45$ $\left(2 \mathrm{H}, \mathrm{d}, J_{4,3 \mathrm{a}}=10.0 \mathrm{~Hz}, \mathrm{H}-4\right), 5.26\left(2 \mathrm{H}, \mathrm{dd}, J_{3,3 \mathrm{a}}=3.5, J_{3,2}=\right.$ $5.8 \mathrm{~Hz}, \mathrm{H}-3), 5.10\left(2 \mathrm{H}, \mathrm{d}, J_{11 \mathrm{~b}, 3 \mathrm{a}}=8.5 \mathrm{~Hz}, \mathrm{H}-11 \mathrm{~b}\right), 3.70$ $\left(2 \mathrm{H}\right.$, ddd, $\left.J_{3,3 \mathrm{a}}=3.5, J_{3 \mathrm{a}, 11 \mathrm{~b}}=8.5, J_{3 \mathrm{a}, 4}=10.0 \mathrm{~Hz}, \mathrm{H}-3 \mathrm{a}\right)$; ${ }^{13} \mathrm{C}-\mathrm{NMR}$ (DMSO-d $\left.{ }_{6}\right): \delta 178.20(\mathrm{C}-6), 156.92$ (C-10a), $138.08\left(\mathrm{C}-4^{\prime \prime}\right), 137.01\left(\mathrm{C}-1^{\prime \prime}\right), 134.92(\mathrm{C}-9), 130.61$ (C-11a), $129.62(\mathrm{C}-7), 128.52\left(\mathrm{C}-3^{\prime \prime}, 5^{\prime \prime}\right), 128.00\left(\mathrm{C}-2^{\prime \prime}, 6^{\prime \prime}\right), 127.7$ (C-2), 124.61 (C-3), 123.42 (C-8), 122.80 (C-6a), 116.08 (C-10), 82.08 (C-4), 47.18 (C-3a), 33.52 (C-11b); MS (ESI): $\mathrm{m} / \mathrm{z}(\mathrm{M})^{+}=667$; Anal Calc. for $\mathrm{C}_{40} \mathrm{H}_{26} \mathrm{O}_{6} \mathrm{~S}_{2}: \mathrm{C}, 72.07 ; \mathrm{H}$, 3.90, S, 9.61; found C, 72.09; H, 3.87; S, 9.59\%.

A.12. Synthesis of 7. Pale yellow solid; yield 21\%; m.p.: $197-$ $199^{\circ} \mathrm{C}$; $\mathrm{IR}(\mathrm{KBr}) \mathrm{cm}^{-1}: 1650(\mathrm{C}=\mathrm{O}) ;{ }^{1} \mathrm{H}-\mathrm{NMR}(400 \mathrm{MHz}$, DMSO- $\left._{6}\right): \delta 8.18\left(2 \mathrm{H}, \mathrm{dd}, J_{\mathrm{p}, \mathrm{o}}=1.5,8.1 \mathrm{~Hz}, \mathrm{H}-7\right), 7.72$ $\left(2 \mathrm{H}, \mathrm{ddd}, J_{\mathrm{p} . \mathrm{m}, \mathrm{o}}=0.9,2.1,8.2 \mathrm{~Hz}, \mathrm{H}-9\right), 7.62\left(2 \mathrm{H}, \mathrm{d}, J_{2,3}=\right.$ $6.0 \mathrm{~Hz}, \mathrm{H}-2), 7.59\left(4 \mathrm{H}, \mathrm{d}, J_{\mathrm{o}}=8.2 \mathrm{~Hz}, \mathrm{H}-3^{\prime \prime}, 5^{\prime \prime}\right), 7.54(6 \mathrm{H}$, m, H-10, $\left.2^{\prime \prime}, 6^{\prime \prime}\right), 7.48\left(2 \mathrm{H}, \mathrm{d}, J_{\mathrm{o}}=8.3 \mathrm{~Hz}, \mathrm{H}-8\right), 6.92(2 \mathrm{H}, \mathrm{d}$, $\left.J_{3,2}=6.0 \mathrm{~Hz}, \mathrm{H}-3\right), 5.72(2 \mathrm{H}, \mathrm{s}, \mathrm{H}-4) ;{ }^{13} \mathrm{C}-\mathrm{NMR}\left(\right.$ DMSO-d $\left.\mathrm{d}_{6}\right)$ : $\delta 178.92$ (C-6), 155.58 (C-10), 141.18 (C-3), 140.92 (C-4), $139.72\left(\mathrm{C}-4^{\prime \prime}\right), 137.41\left(\mathrm{C}-1^{\prime \prime}\right), 135.02(\mathrm{C}-9), 134.82(\mathrm{C}-2)$, 132.18 (C-11b), 131.12 (C-11a), 128.12 (C-3", 5"), 127.42 (C-2" 6" $\left.^{\prime \prime}\right), 124.82$ (C-7), 123.08 (C-3), 122.82 (C-8), 115.98 (C-10), 73.58 (C-4); MS (ESI): m/z (M) ${ }^{+}=663$; Anal Calc. for $\mathrm{C}_{40} \mathrm{H}_{22} \mathrm{O}_{6} \mathrm{~S}$ : C, 72.50; H, 3.32; $\mathrm{S}, 9.66$; found $\mathrm{C}, 72.48 ; \mathrm{H}$, $3.30 ; \mathrm{S}, 9.64 \%$.

A.13. Synthesis of $\mathbf{6} \boldsymbol{a}$. The bischromone $\mathbf{2}$ e provided starting compound 2e and one new photoproduct $\mathbf{6 a}$ in THF under similar condition as used earlier for $\mathbf{2 a}$.

Light yellow solid; yield $28 \%$; m.p.: $251-252^{\circ} \mathrm{C}$; $\mathrm{IR}(\mathrm{KBr}) \mathrm{cm}^{-1}: 1649(\mathrm{C}=\mathrm{O}) ;{ }^{1} \mathrm{H}-\mathrm{NMR}(400 \mathrm{MHz}, \mathrm{DMSO}-$ $\left.\mathrm{d}_{6}\right): \delta 8.20\left(2 \mathrm{H}, \mathrm{dd}, J_{\mathrm{p}, \mathrm{o}}=1.5,8.1 \mathrm{~Hz}, \mathrm{H}-7\right), 7.74(2 \mathrm{H}, \mathrm{ddd}$, $\left.J_{\mathrm{p}, \mathrm{m}, \mathrm{o}}=1.4,2.3,7.9 \mathrm{~Hz}, \mathrm{H}-9\right), 7.58\left(10 \mathrm{H}, \mathrm{m}, \mathrm{H}-10,2^{\prime \prime}, 3^{\prime \prime}\right.$, $\left.5^{\prime \prime}, 6^{\prime \prime}\right), 7.48\left(2 \mathrm{H}, \mathrm{d}, J_{o}=8.0 \mathrm{~Hz}, \mathrm{H}-8\right), 6.43(2 \mathrm{H}, \mathrm{brs}, \mathrm{H}-2)$, $5.48\left(2 \mathrm{H}, \mathrm{d}, J_{4,3 \mathrm{a}}=11.0 \mathrm{~Hz}, \mathrm{H}-4\right), 5.20\left(2 \mathrm{H}, \mathrm{d}, J_{3,3 \mathrm{a}}=1.0\right.$, $\left.J_{3,2}=2.7 \mathrm{~Hz}, \mathrm{H}-3\right), 5.06\left(2 \mathrm{H}, \mathrm{d}, J_{11 \mathrm{~b}, 3 \mathrm{a}}=8.2 \mathrm{~Hz}, \mathrm{H}-11 \mathrm{~b}\right), 3.67$ $\left(2 \mathrm{H}, \mathrm{ddd}, J_{3 \mathrm{a}, 3}=1.0, J_{3 \mathrm{a}, 11 \mathrm{~b}}=8.8, J_{3 \mathrm{a}, 4}=11.0 \mathrm{~Hz}, \mathrm{H}-3 \mathrm{a}\right)$; ${ }^{13} \mathrm{C}-\mathrm{NMR}$ (DMSO- $\mathrm{d}_{6}$ ): $\delta 177.68$ (C-6), 156.88 (C-10a), $136.92\left(\mathrm{C}-4^{\prime \prime}\right), 136.01$ (C-1"), 134.82 (C-9), 130.52 (C-11a), 129.58 (C-7), 128.48 (C-3", $\left.5^{\prime \prime}\right), 128.11\left(\mathrm{C}-2^{\prime \prime}, 6^{\prime \prime}\right), 127.68$ (C-2), 124.58 (C-3), 123.38 (C-8), 122.72 (C-6a), 116.12 (C-10), 82.10 (C-4), 47.12 (C-3a), 33.48 (C-11b); MS (ESI): $\mathrm{m} / \mathrm{z}(\mathrm{M})^{+}=635$; Anal Calc. for $\mathrm{C}_{40} \mathrm{H}_{26} \mathrm{O}_{8}$ : C, 75.70; $\mathrm{H}$, 4.10; found C, 75.92; H, 4.11\%.

\section{Acknowledgment}

Authors are highly thankful to CSIR, New Delhi for providing the grant for this research work in the form of CSIR, JRF.

\section{References}

[1] A. Wojcicki and C. E. Shuchart, "Transiton-metal-propargyl complexes: versatile reagents in synthesis," Coordination Chemistry Reviews, vol. 105, pp. 35-60, 1990.

[2] I. Fleming, "2.2-allylsilanes, allylstannanes and related systems," Comprehensive Organic Synthesis , vol. 2, pp. 563-593, 1991.

[3] A. S. Gupta, B. S. Prabhu, and M. S. Phull, "Synthesis of 3-amino-(N-aryl substituted)-6-bromo-2H-1-benzopyran-2ones and 6-bromo-3-phenoxy substituted-2H-1-benzopyran2-ones as potential antitubercular agents-part I," Indian Journal of Chemistry Section B, vol. 35, no. 2, pp. 170-171, 1996.

[4] R.-S. Liu, "Synthesis of oxygen heterocycles via alkynyltungsten compounds," Pure and Applied Chemistry, vol. 73, no. 2, pp. 265-269, 2001.

[5] B. Schmidt, "An olefin metathesis/double bond isomerization sequence catalyzed by an in situ generated ruthenium hydride species," European Journal of Organic Chemistry, no. 5, pp. 816-819, 2003.

[6] M. Fan, Z. Yan, W. Liu, and Y. Liang, "DABCO-catalyzed reaction of $\alpha$-halo carbonyl compounds with dimethyl acetylenedicarboxylate: a novel method for the preparation of polysubstituted furans and highly functionalized $2 \mathrm{H}$-pyrans," Journal of Organic Chemistry, vol. 70, no. 20, pp. 8204-8207, 2005.

[7] H. Menz and S. F. Kirsch, "Synthesis of stable 2H-pyran5 -carboxylates via a catalyzed propargyl-claisen rearrangement/oxa-6 $\pi$ electrocyclization strategy," Organic Letters, vol. 8, no. 21, pp. 4795-4797, 2006.

[8] F. Požgan, K. Kranjc, V. Kepe, S. Polanc, and M. Kočevar, "Synthesis of 2H-pyran-2-ones and fused pyran-2-ones as useful building blocks," Arkivoc, vol. 2007, no. 8, pp. 97-111, 2007.

[9] S. A. Worlikar, T. Kesharwani, T. Yao, and R. C. Larock, "Synthesis of 3,4-disubstituted 2H-benzopyrans through CC bond formation via electrophilic cyclization," Journal of Organic Chemistry, vol. 72, no. 4, pp. 1347-1353, 2007.

[10] Y. K. Chung and G. C. Fu, "Phosphine-catalyzed enantioselective synthesis of oxygen heterocycles," Angewandte Chemie, vol. 48, no. 12, pp. 2225-2227, 2009.

[11] M. McConville, J. Ruan, J. Blacker, and J. Xiao, "Synthesis of oxygen heterocycles by regioselective Heck reaction," Organic 
and Biomolecular Chemistry, vol. 8, no. 24, pp. 5614-5619, 2010.

[12] F. M. Saleshier, S. Suresh, N. Anita, Jubiakarim, and M. C. Diwakar, "Design, docking and synthesis of some 6benzimidazoyl pyrans and screening of their anti tubercular activity," European Journal of Chemistry, vol. 1, no. 2, pp. 150159, 2011.

[13] W. M. Horspool, Photochemistry in Organic Synthesis, Royal Society of Chemistry, London, UK, 1986.

[14] H. A. J. Carless and S. Mwesigye-Kibende, "Intramolecular hydrogen abstraction in ketone photochemistry: the first examples of $\zeta$-hydrogen abstraction," Journal of the Chemical Society, Chemical Communications, no. 21, pp. 1673-1674, 1987.

[15] P. J. Wagner, "1,5-Biradicals and five-membered rings generated by delta-hydrogen abstraction in photoexcited ketones," Accounts of Chemical Research, vol. 22, pp. 83-91, 1989.

[16] G. A. Kraus and Y. Wu, "1,5- and 1,9-hydrogen atom abstractions. photochemical strategies for radical cyclizations," Journal of the American Chemical Society, vol. 114, no. 22, pp. 8705-8707, 1992.

[17] G. A. Kraus, W. Zhang, and Y. Wu, "1,12-Hydrogen atom abstraction reactions of $\alpha$-keto esters," Chemical Communications, no. 21, pp. 2439-2440, 1996.

[18] K. K. Park, I. K. Han, and J. W. Park, "Photochemical synthesis of cyclophanes containing tethered benzofuran rings," Journal of Organic Chemistry, vol. 66, no. 20, pp. 6800-6802, 2001.

[19] S. C. Gupta, A. Saini, S. Sharma, M. Kapoor, and S. N. Dhawan, "A photochemical synthesis of spiropyrans," Tetrahedron Letters, vol. 37, no. 49, pp. 8913-8916, 1996.

[20] T. Sumathi and K. K. Balasubramanian, "A photochemical route to 2-alkenyl- and 2-ethynylbenzofurans," Tetrahedron Letters, vol. 31, no. 26, pp. 3775-3778, 1990.

[21] M. Yusuf and R. Kumar, "Synthesis and photochemical Habstractions of some 2-thienyl/furyl-.- allyloxybischromones," Indian Journal of Chemistry Section B, vol. 49, no. 2, pp. 216223, 2010.

[22] M. Yusuf and R. Kumar, "Photolysis of some 2-butenyl/ butynylbischromones: effect of solvent polarity," Organic Communications, vol. 2, no. 1, pp. 7-19, 2009.

[23] M. Yusuf, R. Kumar, and S. C. Gupta, "Photochemical transformations of 1,10-decylbischromones: effect of spacer length," Arkivoc, vol. 2006, no. 15, pp. 28-36, 2006.

[24] S. C. Gupta, A. Saini, D. Kumar et al., "On the mechanism for the phototransformation of 3-alkoxy-2-(2'-furyl) -4-oxo$4 \mathrm{H}-1$-benzopyrans," Journal of the Chemical Society, Perkin Transactions 1, no. 2, pp. 177-181, 1995.

[25] S. C. Gupta, S. Sharma, A. Saini, and S. N. Dhawan, "Photochemistry of chromones; photoreorganisation of 3-alkoxy2-thienyl-4-oxo-4H-1-benzopyrans," Journal of the Chemical Society, Perkin Transactions 1, vol. 1, pp. 2391-2395, 1999.

[26] N. S. Yadav, S. N. Dhawan, and S. C. Gupta, "Photoirradiation of some 3-benzyloxy-2-phenyl-4-oxo-4H-1benzopyrans," Journal of the Indian Chemical Society, vol. 67, no. 9, pp. 770-772, 1990.

[27] K. S. Pandey and N. Khan, "Synthesis and antimicrobial study of novel 1-aryl-2-oxo-indano[3,2-d] pyrido/pyrimido[1,2b] pyrimidines," Archiv der Pharmazie, vol. 341, no. 7, pp. 418423, 2008. 


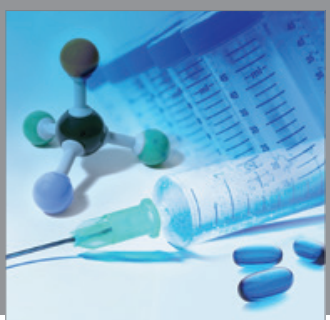

International Journal of

Medicinal Chemistry

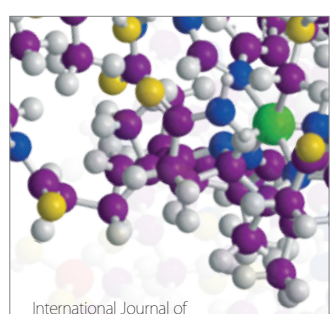

Carbohydrate Chemistry

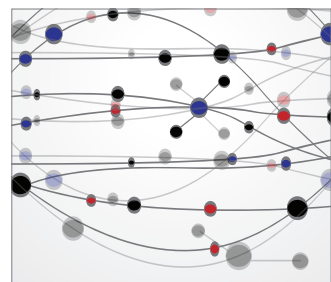

The Scientific World Journal
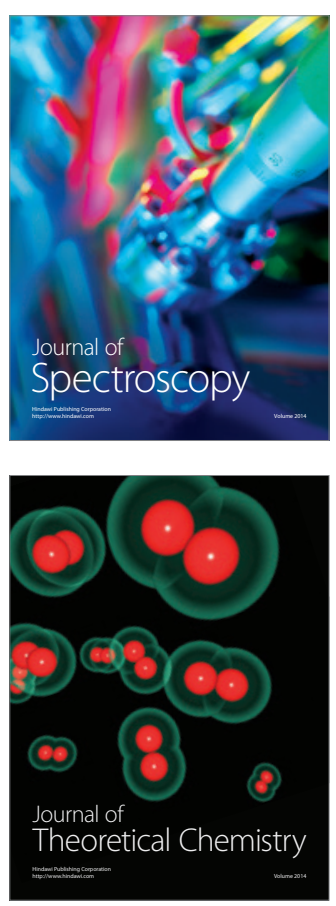
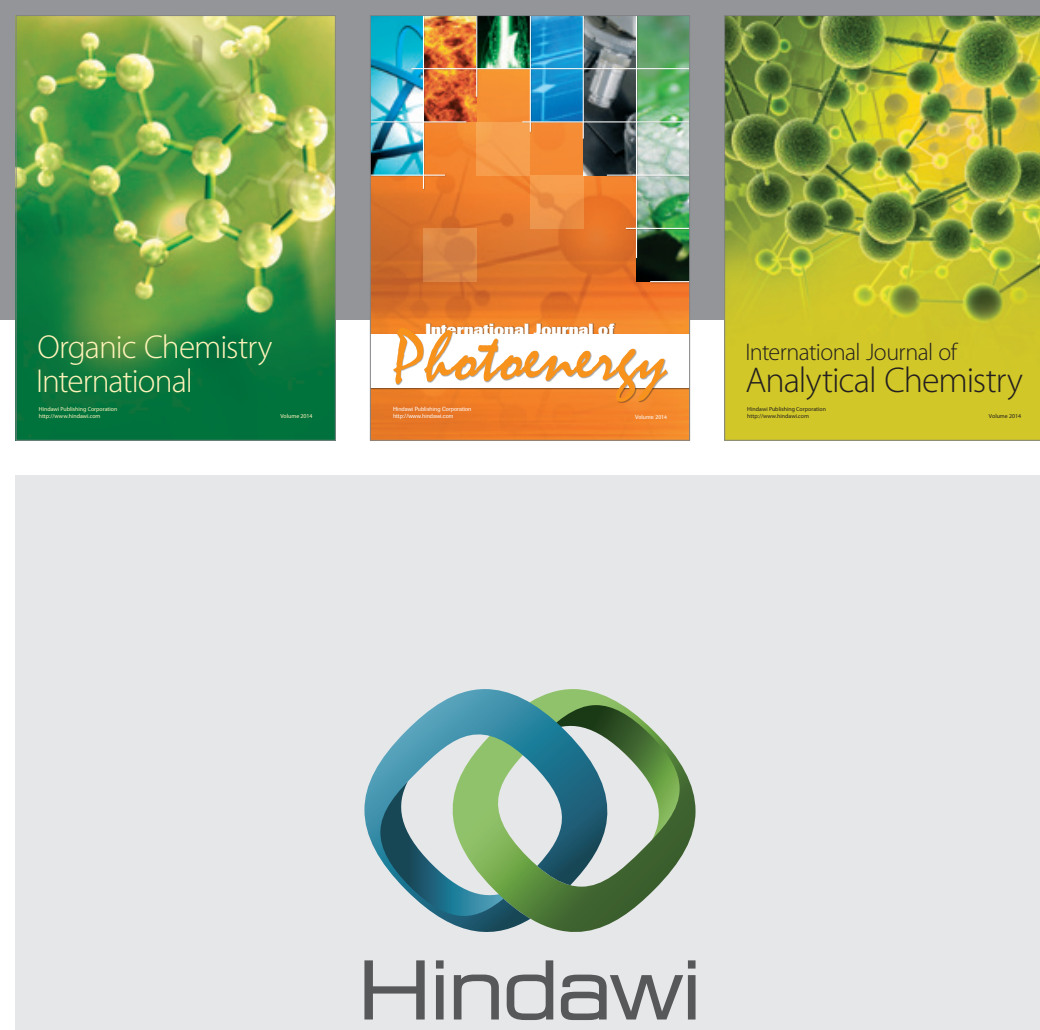

Submit your manuscripts at

http://www.hindawi.com
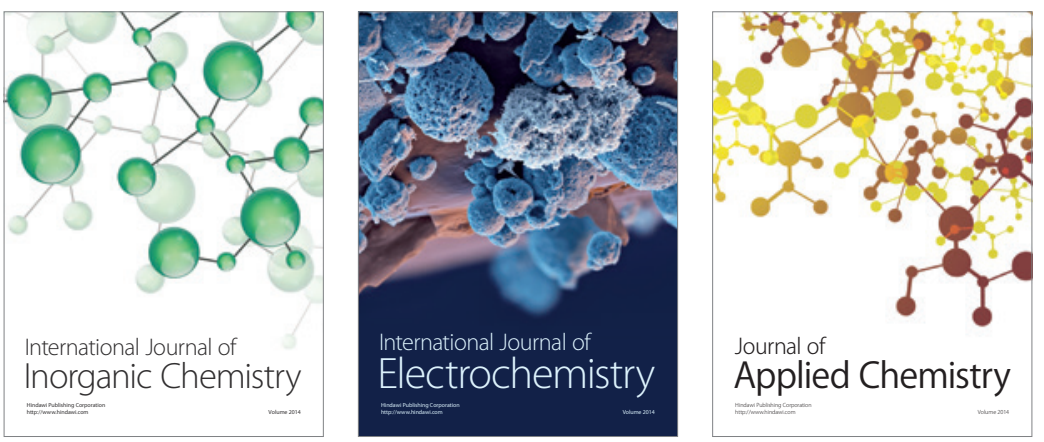

Journal of

Applied Chemistry
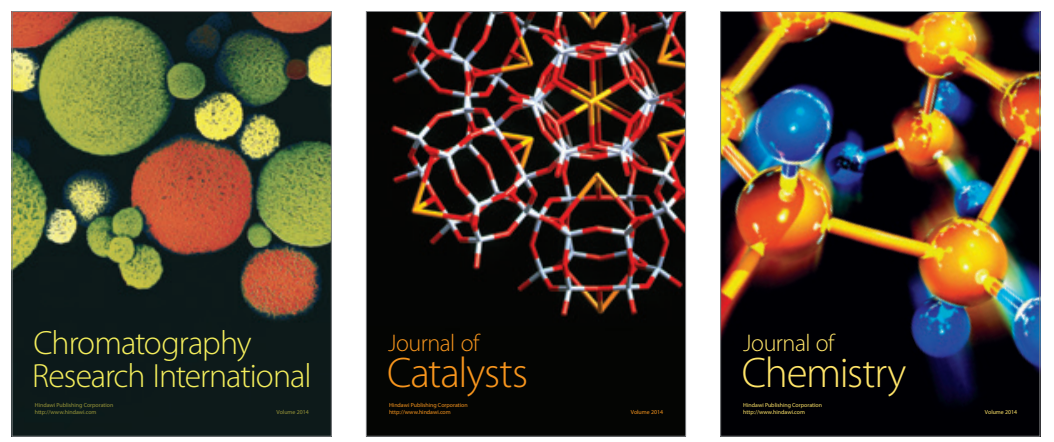
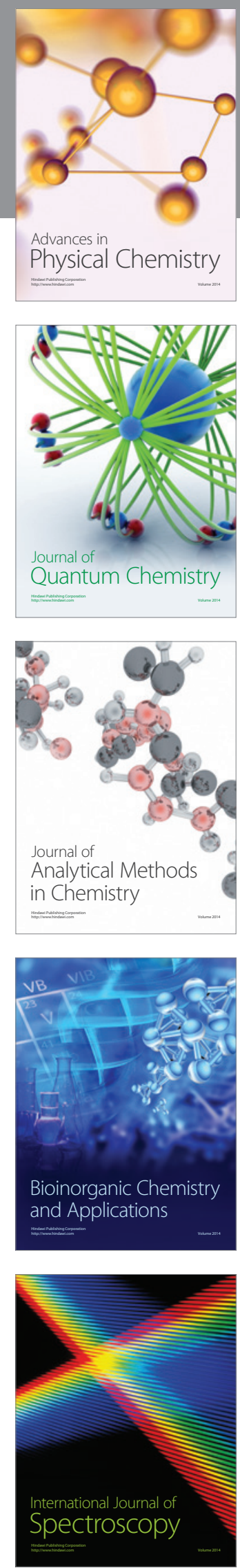